\title{
El estímulo de la motivación intrínseca del estudiantado en un curso de inglés como lengua extranjera
}

The stimulation of the intrinsic motivation of students in an English as a foreign language course

Ruth Cristina Hernández Ching ${ }^{1}$ Damaris Cordero Badilla² Universidad Nacional de Costa Rica

Recibido: 11.12 .2020

Aceptado: 26.12.2020

\section{Resumen}

Se comparte la experiencia de una investigación mixta en un curso de inglés integrado I para otras carreras en una universidad pública costarricense mediante el uso de los avatares como estrategia lúdica para la enseñanza. Se aplican dos enfoques socio-cognitivistas comunicativos: la teoría genética de Piaget y la sociocultural de Vygotsky. Se pretende dar respuesta a la pregunta generadora: ¿Existe una incidencia con el uso de avatares para estimular la motivación intrínseca del estudiantado universitario en el proceso de enseñanza del inglés como lengua extranjera? Se trabajó con una población finita de 29 estudiantes, con edades comprendidas entre los 18 a 25 años, 15 mujeres y 14 varones. Se utilizaron como

\footnotetext{
${ }^{1}$ ruth.hernandez.ching@una.cr

http://orcid.org/0000-0002-3002-7496

2 damaris.cordero.badilla@una.cr https://orcid.org/0000-0001-5556-176X
} 
instrumentos de medición una rúbrica de criterios de coevaluación del recurso educativo multimedia, un cuestionario de prueba MAPE II de pregunta cerrada (Sí-No) y dos rúbricas de apropiación cognitiva, una antes y otra después de la aplicación de la propuesta. Aunque la experiencia no es representativa dada la cantidad de cursos del mismo nivel que se impartió durante el semestre, el experimento, a pequeña escala, demostró que los estudiantes incrementaron su nivel cognitivo del tema propuesto al trabajar con una estrategia tecnológica tal como VOKI y de manera grupal.

Palabras clave: avatar, motivación intrínseca, adquisición de lenguas extranjeras, aprendizaje híbrido, sociocognitivismo.

\begin{abstract}
This article aims to share a mixed investigation in an Integrated English I for other major course in a Costa Rican public university by using avatars as a ludic tool for teaching English as a foreign language. Sociocognitive communicative approaches, including Piaget`s genetic theory and Vygotsky's sociocultural one, are used to formulate the instructional design of this proposal. The following generating question is addressed: Is there an incidence by using avatars to stimulate university students intrinsic motivation of EFL? For this quantitative investigation, the use of a multimedia teaching resource co-evaluation criteria scale, a yes-no question test, and a cognitive appropriation rubric, before and after the proposal, are used. While it is true that the experience is not representative within the number of courses of the same level that was taught during that semester, the experiment, on a small scale, showed that students increased their cognitive level of the proposed topic by working with VOKI as technological strategy and in groups.
\end{abstract}

Keywords: avatar - intrinsic motivation - foreign language acquisition - blended learning sociocognitivism 


\section{Introducción}

La idea para esta propuesta de investigación nace de la necesidad que tienen muchos estudiantes con el reto de aprender inglés en un contexto universitario. Son estudiantes que se matriculan en espacios de enseñanza formal en los cuales reciben aproximadamente 7 horas por semana de clase, y la cantidad de contenido o input al que están expuestos está programado a cubrir las cuatro microdestrezas básicas (lectura, escritura, escucha y conversación). Por otro lado, al ser en su mayoría estudiantes adultos que algunas veces trabajan, tienen familia con hijos, poseen adecuaciones curriculares y que provienen de contextos educativos con baja formación académica o con una situación socioeconómica desfavorable, el esfuerzo que realizan en casa para estudiar el idioma por lo general no es el adecuado. Por lo tanto, estos aprendices requieren de material de apoyo extracurricular y una guía para el estudio en casa.

La idea del uso de las herramientas educativas en línea es desarrollar un espacio de apoyo en el cual los estudiantes que están registrados en Inglés Integrado I tengan a su alcance un instrumento de fácil acceso para repasar los contenidos que se cubren en clase y para conocer de forma específica sobre las actividades a realizar y las áreas de esta lengua que se refuerzan en cada actividad. Si los profesores que imparten estos cursos brindan a sus estudiantes la oportunidad de hacer repasos breves de los contenidos que se desarrollan en clase en este formato virtual, ellos podrán abrir un espacio prometedor de logros en cuanto a motivación y optimización en el uso del tiempo tanto para los profesores como para los estudiantes y así, a largo plazo, evitar resultados negativos en el aprendizaje permanente, evaluaciones y desempeño.

\section{Revisión de la literatura}

Se realizó la lectura de una serie de autores que han investigado y propuesto proyectos en el área de la educación mediada a través de recursos tecnológicos. Como resultado de esta exploración, se puede decir que un docente contemporáneo al realizar una práctica educativa que va de la mano con las nuevas tendencias (respetando todo el saber construido a lo largo 
del tiempo en el área educativa), es un profesional que no sólo investiga y conoce nuevas formas de tecnología y sus aplicaciones a la enseñanza de su especialidad, sino que es a la vez un creador de sus propios espacios y productos de orientación virtual por medio de recursos tecnológicos o sin ellos. La revolución tecnológica nos obliga a formar parte de esa aldea global virtual de la cual la educación no se escapa, sin embargo, tal y como lo recomienda Tejada (2000) en su artículo, debemos siempre de buscar el mejor equilibrio y usar la tecnología según las necesidades de los estudiantes, de la realidad que ofrece su contexto educativo y de los objetivos y contenidos que componen el curso a desarrollar.

Existe un trabajo de licenciatura realizado por Vega (2004) en donde se expone el valor de las actividades lúdicas en los procesos de enseñanza de los tiempos verbales en la educación secundaria. En el 2016, Hernández-Ching y Esquivel realizaron una investigación-acción con el propósito de reflejar la experiencia de aprendizaje de los estudiantes de la carrera de Bachillerato en Informática y Tecnología Multimedia de la Universidad de Costa Rica, Sede del Pacífico, cuando se les consultó por el uso de estrategias de aprendizaje del inglés como segunda lengua al usar videojuegos en inglés. También se encuentra el trabajo de Cordero, D., Hernández-Ching y Tamargo, R. (2018) en donde se comparten experiencias enriquecedoras que fortalecen las competencias lingüísticas en estudiantes de inglés como lengua extranjera con el uso de aplicaciones informáticas y donde se documenta el uso de avatares con el uso de Voki también.

Para este trabajo de investigación se utilizaron dos enfoques, el enfoque inductivo, en donde a los estudiantes, en parejas, se les asignó un tema gramatical para que investigarán, en horas de estudio extraclase, sus reglas y posteriormente, las expusieron al grupo por medio de un avatar, propusieran a sus compañeros ejercicios de resolución de problemas y resolvieran dudas. En una segunda etapa de la exposición, el resto del grupo realizó una coevaluación del trabajo de cada pareja (anexo 3). Al estudiantado se le presentó como propuesta de investigación el uso de las reglas gramaticales de la conjugación del verbo simple presente en tercera persona, en oraciones positivas, negativas y preguntas. Se trabajó con una población de 29 estudiantes, que se subdividió en parejas y un grupo de tres miembros. 
Para lograr los actos pedagógicos en cuestión, los estudiantes deben expandir su vocabulario, y así, entender los textos que leen y escriben, es decir, adquirir la terminología para adaptarla a las situaciones comunicativas, ya que, como lo concibe Saborío (2017), el lenguaje es un ente de interacción social, a través de funciones, interacciones, negociaciones y situaciones. A pesar de que la conjugación verbal con los verbos activos tiende a ser considerado más un error de producción conversacional, diferentes autores lo consideran un tipo de error común en la escritura de inglés como segunda lengua (Azar, 2000, Córdoba y Solís, 2015, y Ferris, 2011).

Los aportes de la enseñanza de la lengua asistida por medio de las computadoras a mediados de la década de los años de 1990 incorporan el componente de la interactividad e interacción lo cual resulta beneficioso para la enseñanza de segundas lenguas. Autores como Krashen (1982) han tenido influencia en esta materia pues otorgan un valor significativo a los procesos comunicativos más allá de los formales (gramaticales), pues para él, lo formal se va adquiriendo de manera inherente conforme el input sea significativo.

¿Con este preámbulo en mente, se realizó esta investigación de carácter exploratorio para tratar de establecer una incidencia entre el uso de avatares para estimular la motivación intrínseca de los estudiantes universitarios en el proceso de enseñanza de la gramática de inglés como segunda lengua? Esta investigación de tipo cuantitativo utilizó como instrumentos de medición una escala de criterios de evaluación del recurso educativo multimedia, un cuestionario de prueba MAPE II de pregunta cerrada Sí-No y dos rúbricas de apropiación cognitiva, una antes y otra después de la aplicación de la propuesta. A continuación, se comparte la metodología empleada. 


\section{La motivación intrínseca}

La motivación intrínseca es aquella descrita por Sáez (2018) como la "que nos impulsa a hacer cosas por el simple gusto de hacerlas, y es la que nace en el propio individuo" (p. 1). Es aquella que se basa en la autonomía y competencia, la satisfacción y el disfrute (Larrenua, 2014), y un elemento importante de considerar cuando se analiza el aprendizaje de un estudiante de lengua, al ser las emociones excelentes estímulos para el logro durante el proceso de la adquisición y el aprendizaje de un idioma y para su correcto desarrollo (Larrenua, 2014, p. 5).

El uso de estrategias de motivación tales como los avatares en una clase de inglés, ayuda a que las lecciones sean amenas y a que se cree un ambiente propicio de aprendizaje, en el cual el estudiantado mantiene gran interés por aprender el idioma, ya que se convierte en el protagonista de la acción, se le fortalece su sensación de agrado, de competencia y de imagen positiva hacia lo que hace" (Galeano, 2016, p. 1).

\section{EI Voki como recurso educativo}

VOKI es un recurso educativo abierto, el cual es definido como un tipo de aplicación informática de tipo widget o pequeño programa. Con esta aplicación, se crea un personaje o avatar que se mueve y habla según el texto que se introduce en una ventana de diálogo, y este pasa de texto a voz. La palabra avatar es definida por la Real Académica como una reencarnación o transformación, y por Carretero Oyarzún y Aizpurua y Ortiz (2004), como representaciones virtuales con del uso de imágenes, hologramas o dibujos con animaciones y sonido; dicha representación le permite al humano coexistir y expresarse tanto en el mundo cibernético como el real.

VOKI es una aplicación de mucha utilizada en una clase de idiomas al servir de ayuda para que los disidentes mejoren sus competencias lingüísticas en escritura y en la pronunciación de las palabras de forma lúdica. Es un programa que presenta el contenido de forma visual o 
auditiva, la vez que estimula la creatividad, la imaginación y la incorporación de las TIC en el aula.

VOKI permite presentar contenidos académicos multimedia de cualquier índole, en especial texto y voz. La herramienta resulta atractiva para los estudiantes pues el que presenta la información es un avatar personalizado al gusto del estudiante. Después de ofrecer la explicación de cómo ingresar y utilizar este sitio de avatares, el profesor puede motivar a los estudiantes para que utilicen VOKI con la creación de un personaje. Este sería un avatar al cual se le escribirá un diálogo, con el uso de estructuras gramaticales, sus propias investigaciones y temas estudiados durante las lecciones.

La investigación teórica de esta propuesta, se concibió al elaborar un prototipo de estrategia docente por medio del trabajo colaborativo entre cinco integrantes del curso Tecnología Educativa Aplicada en la Enseñanza de una Lengua Extranjera impartido en una universidad pública durante un cuatrimestre del 2014 (ver anexo 1), y en el III ciclo del año 2017, para la etapa de acción se tomó una de las actividades propuestas en dicha estrategia, el uso de avatares, para realizar la investigación en otra universidad pública.

En esta oportunidad, se trabajó con una población finita de 29 estudiantes, 15 mujeres y 14 hombres con edades comprendidas entre los 18 y 25 años quienes cursan diferentes carreras como archivística, economía, enseñanza de las ciencias, enseñanza de las matemáticas, relaciones internacionales, entre otras. Para ello se aplicaron dos tipos de enfoques socio cognitivistas comunicativos, con la teoría genética de Piaget y la sociocultural de Vygotsky. Con ello se buscó dar respuesta a la siguiente pregunta generadora:

¿Existe una incidencia con el uso de avatares para estimular la motivación intrínseca de los estudiantes universitarios en el proceso de aprendizaje de la escritura y el habla en inglés como segunda lengua? Esta investigación de tipo cuantitativo utilizó como instrumentos de medición una escala de criterios de evaluación del recurso educativo multimedia (anexo 3) diseñado como parte de la elaboración del prototipo de estrategia docente, un cuestionario de 
prueba MAPE II (motivación hacia el aprendizaje y la ejecución) (anexo 4) de pregunta cerrada Sí-No y dos rúbricas de apropiación cognitiva, una antes y otra después de la aplicación de la propuesta (anexo 2).

\section{Metodología}

Para realizar la sección de acción de la propuesta se trabajó con un grupo compuesto por 29 estudiantes, 15 mujeres y 14 varones. Antes de que los alumnos realizarán las presentaciones grupales de sus trabajos con VOKI, se utilizó una rúbrica de apropiación cognitiva de 12 ítems. El porcentaje del éxito se expone en el análisis de resultados, el cual se calculó realizando según el promedio obtenido por cada integrante del grupo de manera individual. Así mismo, y con la rúbrica que se comparte en el anexo 3, el estudiantado co evaluó a los pares. Los resultados de estas evaluaciones se comparten en la sección de análisis de resultados.

\section{Análisis de resultados}

El porcentaje del éxito al analizar los resultados de la rúbrica de apropiación cognitiva antes de realizar las exposiciones del tema con el uso de VOKI fue de 56,88 por ciento. Un resultado que se obtenido por cada integrante del grupo de manera individual. Posteriormente a las presentaciones, se volvió a utilizar la misma rúbrica de apropiación cognitiva en donde los estudiantes demostraron un porcentaje de éxito del 73,94 por ciento en comparación con el resultado obtenido en la primera medición. Después de las presentaciones grupales, el estudiantado coevalúo a los demás grupos utilizando la rúbrica de criterios de coevaluación del recurso educativo multimedia. El promedio de resultado de evaluación por estudiante fue de 4.26 por ciento de un 5 por ciento, lo cual demuestra un alto grado de satisfacción respecto a la actividad.

Con respecto a los datos arrojados con la prueba MAPE II (anexo 4), en el cuadro 1, 2 y 3 se comparte la tabulación de datos por porcentajes. En los gráficos 1 y 2, se muestra los resultados de la tabulación por porcentajes tomando como una totalidad del 100\% a los 29 
estudiantes. En el cuadro 1, se muestra la tabulación total de ítems de las dos subescalas de manera porcentual.

\section{Cuadro 1.}

Tabulación de los datos recopilados de manera porcentual y total de la encuesta MAPE II

MOTIVACION INTRINSECA

Las tareas demasiado dificiles las hecho de lado con gusto.

Una vida sin trabajar seria maravillose.

Yo hago, como máximo, lo que se pide; y no mas.

Estaria también contento si no tuviese que trabajar.

Mi rendimiento mejora si espero aiguna recompensa especial por él.

Trabajo ùnicamente para ganarme la vida

Yo me harla cargo de un puesto de responsabilidad aunque no estuviera pagado como debiera.

Me siento inquieto si estoy aiguncs dias sin trabajar.

A mayor responsabilidad de la tarea a realizar, yo exigiria una mayor recompensa.

Con tal de hacer algo, soy capaz de trabejar aunque el pago que se dé a mi trabajo sea a todas luces insuficiente.

Prefiero hacer trabajos que lleven consigo cierta dificultad a hacer trabajps fácles.

Cuanto más dificil se torna una tarea, más me animo a realizarla. sí NO Porcentaje de Si Porcentaje de No

$425 \quad 14 \% \quad 86 \%$

\begin{tabular}{l|l|l|l|}
5 & 24 & $83 \%$ & $83 \%$
\end{tabular}

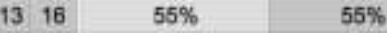

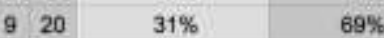

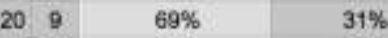

$326 \quad 10 \% \quad 90 \%$

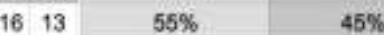

\begin{tabular}{l|l|l|}
16.13 & $55 \%$ & $31 \%$
\end{tabular}

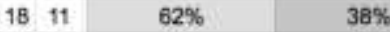

\begin{tabular}{l|l|l|l}
13 & 16 & $45 \%$ & $55 \%$
\end{tabular}

\begin{tabular}{l|l|l|l}
20 & 9 & $69 \%$ & $31 \%$
\end{tabular}

209

Fuente: Elaboración propia.

A continuación, en los cuadros 2 y 3, se comparten los porcentajes obtenidos según aquellos ítems asociados a subescalas que muestran una motivación intrínseca alta o baja:

\section{Cuadro 2.}

Tabulación de los ítems de manera porcentual que puntuaron hacia una motivación intrínseca alta

\begin{tabular}{|c|c|c|c|}
\hline t & MOTIVACION INTRINSECA & Porcentaje de Sí & Porcentaje de No \\
\hline 2 & Las tareas demasiado dificiles las hecho de lado con gusto. & $14 \%$ & $86 \%$ \\
\hline 4 & Una vida sin trabajar serias maraviloss. & $B 3 \%$ & $83 \%$ \\
\hline 5 & Yo hago, como maximo, lo que sa pide; y no mas. & $55 \%$ & $55 \%$ \\
\hline 由 & Estarla tambidn contento si no tuviese que trabajar. & $31 \%$ & $69 \%$ \\
\hline 10 & Mi rendimiento mejora si espero alguna recompensa especial por et. & $69 \%$ & $31 \%$ \\
\hline 14 & Trabajo únicamento para gavarme la vida. & $10 \%$ & $90 \%$ \\
\hline 96 & Para mi es mass importante el poder trabajar que el ganar dinero. & $52 \%$ & $48 \%$ \\
\hline
\end{tabular}

Fuente: Elaboración propia. 


\section{Cuadro 3.}

Tabulación de los ítems de manera porcentual que puntuaron hacia una motivación intrínseca baja
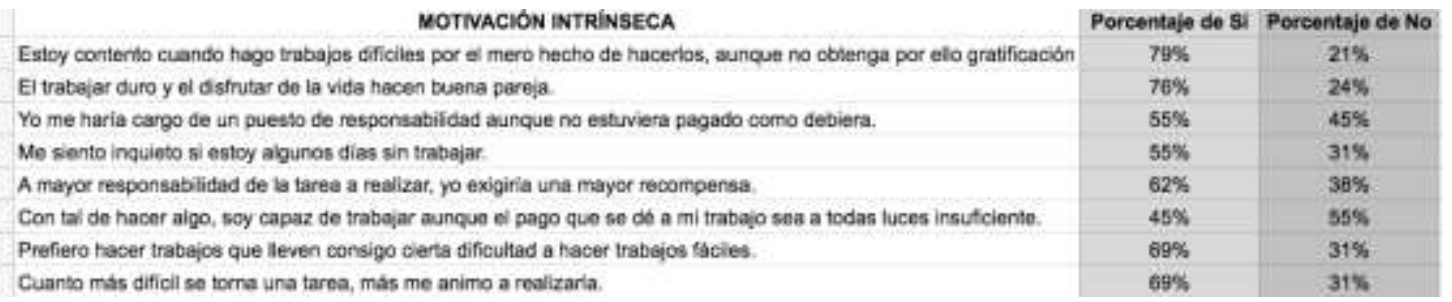

Fuente: Elaboración propia.

\section{Gráfico 1. Subescala 1.}

Subescala de ítems que puntuaron hacia una motivación intrínseca alta

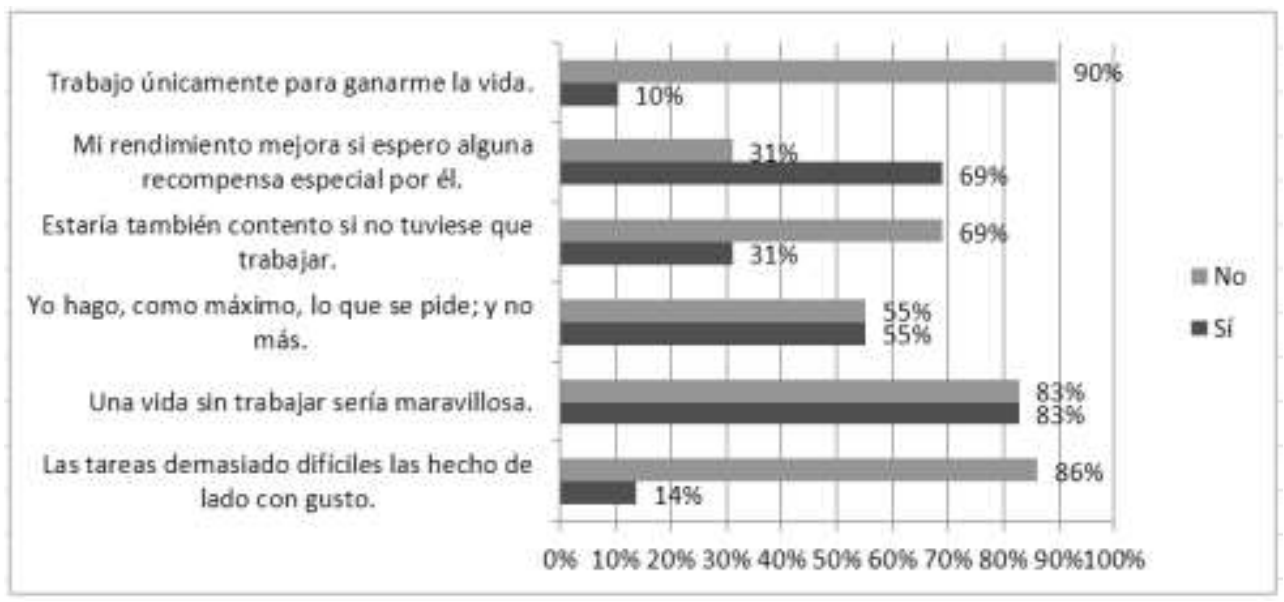

Fuente: Elaboración propia.

Se puede observar que el estudiantado muestra un grado alto de motivación intrínseca en casi todos los ítems. No obstante, se observa que la recompensa sí representa una fuente importante de remuneración como se ilustra en los ítems Mi rendimiento mejora si espero alguna recompensa especial por él (69\% sí, sobre un 31\% de no), y Para mí es más importante el poder trabajar que el ganar dinero ( $52 \%$ sí, sobre un $48 \%$ de no). Por lo tanto, se podría interpretar que la población estudiantil espera, para este caso particular, mayor conocimiento o un excelente resultado en las calificaciones como producto de la participación activa en el proyecto grupal. 


\section{Gráfico 2. Subescala 2.}

Subescala de ítems que puntuaron hacia una motivación intrínseca baja

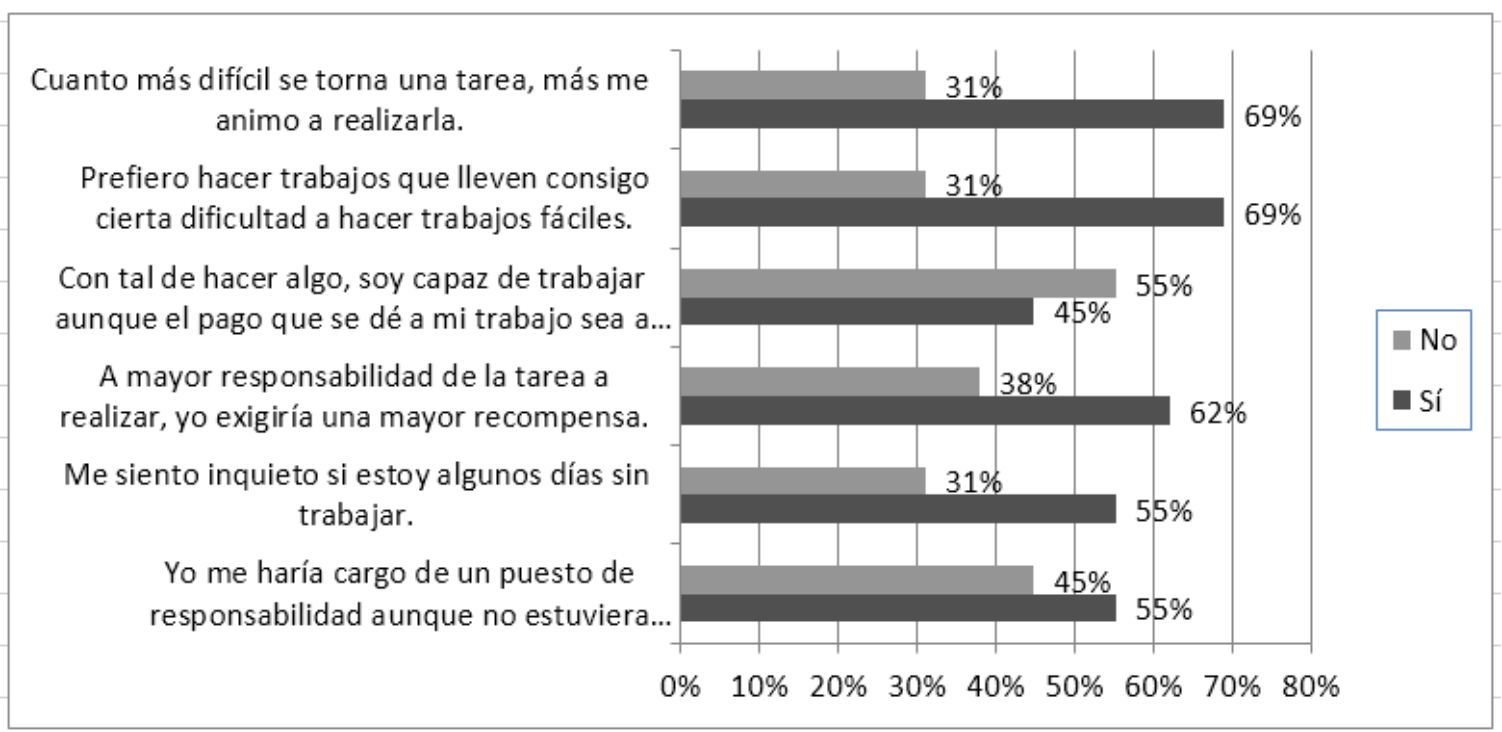

Fuente: Elaboración propia.

Con respecto a los ítems que conducían hacia una motivación intrínseca baja, puede notarse nuevamente motivación alta en el estudiantado, y se observa también que, aunque se harían cargo de un puesto de responsabilidad, aunque no estuviera pagado como debiera, (55\% sí, sobre un $45 \%$ de no), sí esperarían una mayor recompensa conforme a las responsabilidades otorgadas, (62\% sí, sobre un 38\% de no). Por otro lado, la mayoría de las estudiantes determinó que el reto a la hora de realizar las actividades representa una fuente de motivación intrínseca importante, como lo indica el primer ítem del gráfico (69\% sí, sobre un 31\% de no). Nuevamente, estos resultados parecen sugerir, que, a mayor nivel de esfuerzo, los estudiantes esperarían un aprendizaje mayor o una calificación excelente, es decir, una recompensa o remuneración al esfuerzo, para este caso docente, mayor conocimiento, una excelente calificación y el reconocimiento del trabajo realizado por parte de sus pares y docente. Esto hace pensar en las sugerencias de Alessi y Trollip (1985) con respecto a los elementos mencionados anteriormente que podrían desentrañar la motivación intrínseca que existe en cada aprendiz y docente también, y que se construyó de manera conjunta para este caso particular. 


\section{Conclusiones}

Se pueden concluir que el estudio arrojó una incidencia importante entre la motivación intrínseca y la mejora en la apropiación cognitiva con respecto al objetivo de aprendizaje a ser evaluado. No obstante, estos resultados no son concluyentes debido, primero al tamaño de la muestra y al lapso en el que se realizó, y, por otra parte, a que la revisión de la literatura parece indicar que sería ideal proponer al estudiantado pruebas cognitivas comunicativas y no únicamente de medición de aspectos de noción funcional, es decir, por medio, un párrafo, un correo electrónico, un mensaje de texto, en donde se aplique el enfoque comunicativo.

Por otra parte, como también se consultó en la revisión de la literatura, en la enseñanza de los aspectos gramaticales en Costa Rica se dedica una notoria atención a los enfoques inductivos, deductivos, de proceso, y producto, comunicativo y funcional, dejando, muchas veces de lado los enfoques sociocognitivistas. La motivación personal del estudiantado, de la mano con la interacción social de sus compañeros (en donde inclusive tengan la posibilidad de evaluar a sus pares) y docentes parecen mejorar los procesos de aprendizaje. Con este trabajo, se determinó que los enfoques sociocognitivistas para el diseño instruccional de la propuesta presentada por los docentes permitió aumentar los porcentajes de éxito, en los resultados de las pruebas, aún con las limitaciones personales de cada participante que se mencionaron al inicio de este trabajo, lo cual parece indicar ser una opción atractiva para aprender.

El input y la interiorización del encargo pedagógico utilizando estrategias tecnológicas, como los avatares, permitió la interacción y el aprendizaje, no solo con la máquina, sino que también con sus pares y el resto del grupo de trabajo utilizando de por medio el aprendizaje híbrido para aprovechar al máximo el tiempo y las condiciones personales de cada participante del curso. Sin embargo, si bien es cierto existió una participación activa en la construcción de la actividad complementaria, no a todo el estudiantado le gustó la herramienta tecnológica y esto se puede explicar desde la perspectiva de los diferentes estilos de aprendizaje. 


\section{Referencias}

Aguilar, R. A., \& Ramírez-Martinell, A. (2015). La pizarra digital: recurso didáctico para la visualización e interacción académica en educación superior. Ensayos Pedagógicos, 10(2), 149-166. Recuperado de http://www.revistas.una.ac.cr/index.php/ensayospedagogicos/article/view/7683

Alessi S.M. y Trollip S.R. (1985). Computer-based instruction. Methods and Development. Prentice Hall. Nueva Jersey.

Ausubel, D. P. (1968). Educational psychology: a cognitive view. New York: Holt.

Avatar. (2018). Diccionario de la Real Academia Española. Recuperado de http://dle.rae.es/?id=4X6SYjl

Azar, B. (2007). Understanding and using English grammar. New York: Pearson Longman.

Berdugo, M. (2001). Integración de tecnologías multimedia en la enseñanza de lenguas. Lenguaje, 28: 84-107. Recuperado de http://bibliotecadigital.univalle.edu.co/bitstream/10893/2706/3/Rev.Lenguaje\%20No.28.P.8 4-107\%2C2001.pdf

Carretero, M., Oyarzun, D., Aizpurua, I y Ortiz, A. (2004). Animación Facial y Corporal de Avatares 3D a partir de la edición e interpretación de lenguajes de marcas. Asociación VICOMTech. España: Departamento de Educación y Turismo. Recuperado de https://www.researchgate.net/profile/Amalia_Ortiz/publication/228950279_Animacion_Faci al_y_Corporal_de_Avatares_3D_a_partir_de_la_edicion_e_interpretacion_de_lenguajes_de _marcas/links/55642eba08ae9963a11f0433.pdf 
Cataldi, Z. (2000). Metodología de diseño, desarrollo y evaluación de software educativo. Tesis de Magíster en Informática. (versión resumida). Facultad de Informática. UNLP.

Cerezo, L. (2010). Talking to avatars: The computer as a tutor and the incidence of learner`s agency, feedback and grammar for un SLA. Tesis de disertación doctoral de la Universidad de Georgetown. Recuperado de https://repository.library.georgetown.edu/bitstream/handle/10822/558185/CerezoCeballos_g eorgetown_0076D_10997.pdf?sequence=1\&isAllowed=y

Cordero, D., Hernández-Ching, R. y Tamargo, R. (2018). El fortalecimiento de competencias lingüísticas en estudiantes de inglés como lengua extranjera (ILE) con el uso de aplicaciones informáticas. Revista Letras,

Córdoba, P. y Solís, M. (2015). The influence of grammar on English learners`writing accuracy at the School of Modern Languages. Káñina, Rev. Artes y Letras, Univ. Costa Rica, XXXIX(2): 107-121. Recuperado de https://revistas.ucr.ac.cr/index.php/kanina/article/view/21250

Council for Cultural Cooperation Education Committee. (2001). Common European Framework of Reference for Languages: Learning, teaching, assessment. Cambridge: CUP.

Hernández-Ching, R. y Esquivel, A. (2016). Ponencia: Explorando el uso de videojuegos para la adquisición de segundas lenguas en la Universidad de Costa Rica, Sede del Pacífico, VII Jornada de Investigación sobre el Pacífico Costarricense. Puntarenas, Universidad de Costa Rica.

Evans, J. y Lindsay, W. (2005). Administración y control de la calidad. Honduras: Universidad Nacional Autónoma de Honduras. 
Ferris, D. (2011). Treatment of error in second language student writing. Ann Arbor: The University of Michigan.

Galeano, E. (2016). El avatar y la perspectiva lúdica en el aula de ELE: Guadalingo. Editorial Edinumen Blog. Recuperado de http://blog.edinumen.es/index.php/2016/05/25/el-avatar-yel-aula-de-ele-guadalingo/

Galvis, A. (1988). Ambientes de enseñanza-aprendizaje enriquecidos con computador. Boletín de Informática Educativa, 1(2): 117-139.

García, Y., Gradiz, R., Hernández, R., Solano, C., y Turrent, M. (2014). Diseño de prototipo de estrategia docente. Curso Tecnología Educativa Aplicada en la Enseñanza de una Lengua Extranjera. San José: Universidad de Costa Rica.

Gee, J.P. (2004). Learning by design: Games as learning machines. Interactive Educational Multimedia. $\quad 8, \quad 15-23 . \quad$ Recuperado de https://hci.stanford.edu/publications/2006/HowBodiesMatter-DIS2006.pdf

Gutiérrez, C. (2007). Self-evaluation in Learning English as a Foreign Language Through Cooperative Learning Tasks. Memoria de Práctica Dirigida, Universidad de Costa Rica, Costa Rica.

Johnson, D., Maruyama, G., Johnson, R., Nelson, D. \& Skon, L. (1981). The effects of cooperative, competitive and individualistic goal structure on achievement: A meta-analysis. Psychological Bulletin, 89, 47-62.

Klemmer, S., Hartmann, B. \& Takayama, L. (2006). How bodies matter: Five themes for interaction design. DIS 2006, June, 26-28. 
Krashen, S. (1982). Principles and practice in second language acquisition. New York: Prentice Hall.

Larrenua, R. (2014). La motivación en el proceso de enseñanza/aprendizaje de lenguas extranjeras. Memoria del trabajo de final de grado: Universitat de les Illes Balears. Recuperado de http://dspace.uib.es/xmlui/bitstream/handle/11201/145393/Larrenua_Vegara_Roberto.pdf?se quence $=1$

Moreno Martínez, N., Leiva Olivencia, J., \& Matas Terrón, A. (2016). Mobile learning, Gamificación y Realidad Aumentada para la enseñanza-aprendizaje de idiomas. IJERI: International Journal of Educational Research and Innovation, 0(6), 16-34. Recuperado de https://www.upo.es/revistas/index.php/IJERI/article/view/1709/1554

New London Group. (1996). A Pedagogy of Multiliteracies:

Designing Social Futures. Harvard Educational Review, 66, 60-92.

Novak, J. D. \& Cañas A. J. (2008) The Theory Underlying Concept Maps and How to Construct Them, Technical Report IHMC CmapTools 2006-01 Rev 01-2008, Florida Institute for Human and Machine Cognition, available at: http://cmap.ihmc.us/Publications/ResearchPapers/TheoryUnderlyingConceptMaps.pdf.

Perkins, D. (1995). La escuela inteligente. Barcelona: Gedisa.

Pineda, A., Porras, M. y Rodríguez, S (2016). Curso Cooperative Learning Course for English Teaching. Heredia: Universidad Nacional.

Ríos, K. (2014). Curso Tecnología Educativa Aplicada en la Enseñanza de una Lengua Extranjera (FD-6092). San José: Universidad de Costa Rica. 
Saborío, I. (2017). Entrevista sobre los métodos de enseñanza de gramática de segundas lenguas. Heredia: Universidad Nacional.

Saez, F. (2018). Motivacion Intrinseca. Recuperado de https://facilethings.com/blog/es/intrinsic-motivation

Snow, M. and Evans, N. (2013). Principles and Practices for Response in Second Language Writing. Developing Self-Regulated Learners. New York: Routledge.

Tapia, J. y Huertas, J. (2000). Cuestionario MAPE II. Recuperado de http://studylib.es/doc/5725074/cuestionario-m.a.p.e.-ii-instrucciones-esta-prueb consiste

Tejada, J. (2000). La educación en el marco de una sociedad global: algunos principios y nuevas exigencias. Profesorado. Revista de Currículum y Formación de Profesorado, 4(001), 1-13. Recuperado de http://www.redalyc.org/pdf/567/56751266002.pdf

Tecnologia e Informatica. La Historia de Voki. Recuperado de https://tecnologia-einformatoca-grpjm.webnode.com.uy/news/historia-del-voki/

Trujillo, J. M. (2006). Un nuevo currículum: tecnologías de la información en el aula. Educación y Educadores, 9(1), 1-14. Recuperado de http://www.scielo.org.co/scielo.php?script=sci_arttext\&pid=S0123-12942006000100011

Universidad de Costa Rica (2014). Plataforma virtual Mediación Virtual.

Universidad Nacional (2018). Plataforma virtual Aula Virtual.

Vega, M. (2004). El juego como instrumento en la enseñanza de los tiempos verbales en secundaria. Tesis de Graduación, Universidad Estatal a Distancia, Costa Rica. 
Wilson, M. (2002). Six views of embodied cognition. Psychonomic Bulletin \& Review, 9(4), 625-636. Recuperado de http://www.indiana.edu/ cogdev/labwork/WilsonSix ViewsofEmbodiedCog.pdf

Woolfolk, A. (2006). Psicologia Educativa. 7ed. Pearson Education. Prentice Hall Mexico. 


\section{Anexo 1. Instrucciones para el diseño de la actividad de aprendizaje usando Voki. Actividad complementaria 1}

\section{Recurso multimedia: Voki}

Actividad: Los estudiantes configurarán y escuchan presentación de reglas gramaticales Valor: 5\%

La justificación del uso de Voki es presentar de manera diferente las reglas de conjugación de los verbos en tercera persona en presente. La herramienta resulta atractiva para los estudiantes pues el que presenta la información es un avatar y no el profesor. La herramienta es interactiva.

Después de la presentación de la herramienta, el profesor motiva a los estudiantes para que utilicen la herramienta y que se apropien del personaje explicando por sí mismo la manera en que entendieron las reglas gramaticales a partir de sus propias investigaciones o la información en el libro del texto. La actividad se realizará en parejas. Cada presentación tendrá una duración de 3 a 5 minutos y redactarán un informe impreso de un 1 o 2 páginas sobre las impresiones sobre el uso de la aplicación, el aprendizaje y recomendaciones. 


\section{Anexo 2. Rúbrica de apropiación cognitiva de 17 ítems aplicado antes y después de la presentación con avatares por parte del estudiantado. Tomado de Azar (1989).}

Directions: Write -s/-ES in the blanks where necessary and make any other needed changes in the verb. If the verb does not need -S/-ES, put a slash (/) in the blank.

1. Monkeys climb trees.

2. Do you like to climb trees?

3. Do___ Paul like to cook?

4. Alex like to dance.

5. Mike wash__ his own clothes.

6. Rita go to school at seven.

7. Bees make__ honey.

8. A bee visit__ many flowers in one day.

9. Tina get__ her work done on time.

10. Tina and Pat get___ their work done.

11. Do___ Bill get__ his work done?

12. Eric do__n`t get__ it done on time.

13. David carry__ a briefcase to work.

14. Janet play__ tennis every day.

15. A frog catch__ flies with its tongue.

16. Frogs are small green animals that live__ near water.

17. A turtle is another animal that live___ near water. 
Anexo 3. Rúbrica de criterios de coevaluación del recurso educativo multimedia. Valor: $5 \%$.

\begin{tabular}{|c|c|c|c|c|c|c|}
\hline Criterios & Deficiente & Regular & Bueno & $\begin{array}{l}\text { Muy } \\
\text { bueno }\end{array}$ & Excelente & No aplica \\
\hline 1) Material contextualizado. & & & & & & \\
\hline 2) Acorde con el objetivo. & & & & & & \\
\hline $\begin{array}{l}\text { 3) Diferencias entre los } \\
\text { estudiantes. }\end{array}$ & & & & & & \\
\hline $\begin{array}{l}\text { 4) Presentación de los } \\
\text { contenidos. }\end{array}$ & & & & & & \\
\hline 5) Legibilidad. & & & & & & \\
\hline 6) Diseño atractivo. & & & & & & \\
\hline $\begin{array}{l}\text { 7) Organización de la } \\
\text { información. }\end{array}$ & & & & & & \\
\hline 8) Flexibilidad metodológica. & & & & & & \\
\hline 9) Flexibilidad pedagógica. & & & & & & \\
\hline 10) Aprendizaje colaborativo. & & & & & & \\
\hline
\end{tabular}

Fuente: Hernández-Ching, R., García, Y., Gradiz, R., Solano, C. y Turrent, M. (2014). (Elaboración propia)

\section{Definición de los indicadores}

Deficiente: El material no contiene ninguno de los elementos requeridos.

Regular: El material posee únicamente uno de los elementos requeridos.

Bueno: Cumple con algunos de los elementos requeridos.

Muy bueno: Cumple con la mayoría de los elementos requeridos. 
Excelente: El material posee todos los elementos requeridos.

No aplica: El material no hace alusión los elementos requeridos.

\section{$>$ Explicación de los criterios}

1. Material contextualizado: Contextualizado en el sistema educativo.

2. Acorde con el objetivo: Relacionado con el objetivo de aprendizaje del tema que se está estudiando.

3. Diferencias entre los estudiantes: Contempla los estilos de aprendizaje y capacidades de los estudiantes.

4. Facilita adquisición de contenidos: Facilita la adquisición y profundización de los contenidos pedagógicos propuestos en el programa de estudios.

5. Legibilidad: Es legible, facilitan la lectura y captan la atención del usuario (tamaño de letra, página, colores). La distribución de los diferentes elementos de la página es apropiada.

6. Diseño atractivo: Contiene elementos que lo hacen atractivo e interactivo para los estudiantes (imágenes, sonido y video, uso de color).

7. Organización de la información: Contiene presentación, objetivos, contenidos y actividades.

8. Flexibilidad metodológica: Permite que el estudiante pueda elegir la modalidad de aprendizaje que mejor se adapte a sus necesidades sea ésta virtual, presencial o mixta.

9. Flexibilidad metodológica: Se adapta a diferentes metodologías de aprendizaje.

10. Aprendizaje colaborativo: Facilita el aprendizaje colaborativo entre los estudiantes.

\section{$>$ Comentarios:}


Anexo 4. Cuestionario MAPE II

\begin{tabular}{|c|c|c|}
\hline $\begin{array}{l}\text { INSTRUCCIONES: Esta prueba consiste en una serie de frases que se refieren a ti } \\
\text { mismo y a tu forma de pensar. }\end{array}$ & & \\
\hline Para cada frase existen dos alternativas. & & \\
\hline Si estás de acuerdo con la afirmación, señala, por favor Sí. & & \\
\hline En caso de no estarlo, señala, por favor, NO. & & \\
\hline Sé sincero en las respuestas, no dejes ningún ítem sin contestar. & & \\
\hline & SÍ & NO \\
\hline Las tareas demasiado difíciles las echo de lado con gusto. & & \\
\hline $\begin{array}{l}\text { Estoy contento cuando hago trabajos difíciles por el mero hecho de hacerlos, aunque no } \\
\text { obtenga por ello gratificación alguna. }\end{array}$ & & \\
\hline Una vida sin trabajar sería maravillosa. & & \\
\hline Yo hago, como máximo, lo que se pide; y no más. & & \\
\hline Estaría también contento si no tuviese que trabajar. & & \\
\hline $\begin{array}{l}\text { Yo me haría cargo de un puesto de responsabilidad, aunque no estuviera pagado como } \\
\text { debiera. }\end{array}$ & & \\
\hline Me siento inquieto si estoy algunos días sin trabajar. & & \\
\hline Mi rendimiento mejora si espero alguna recompensa especial por él. & & \\
\hline A mayor responsabilidad de la tarea a realizar, yo exigiría una mayor recompensa. & & \\
\hline $\begin{array}{l}\text { Con tal de hacer algo, soy capaz de trabajar, aunque el pago que se dé a mi trabajo sea a } \\
\text { todas luces insuficiente. }\end{array}$ & & \\
\hline Prefiero hacer trabajos que lleven consigo cierta dificultad a hacer trabajos fáciles. & & \\
\hline Trabajo únicamente para ganarme la vida. & & \\
\hline to más difícil se torna una tarea, más me animo a realizarla. & & \\
\hline
\end{tabular}

Fuente: Hernández-Ching, R., Cordero, D. (2018). (Elaboración propia) 
REVISTA INTERNACIONAL DE PEDAGOGÍA E INNOVACIÓN EDUCATIVA |

Volumen 1. Número 1. Enero - Junio 2021

ISSN: 2745-0341 (En línea) 